\title{
Congratulations for the 30th Anniversary of the Robotics Society of Japan
}

President of the Korea Robotics Society

Dong-Soo Kwon

On behalf of the Korea Robotics Society (KROS), it is a great honor and pleasure for me to celebrate the 30th anniversary of the Robotics Society of Japan (RSJ).

Over the past 30 years, RSJ has contributed enormously in the domestic sphere by promoting robotics research and also in the international sphere by exchanging information and encouraging cooperation worldwide by sponsoring many international conferences and successfully publishing the international journal, "Advanced Robotics." The Advanced Robotics journal has grown steadily and is now a profound international journal that has a wide international readership of researchers and users. The Advance Robotics has been an invaluable tool to inform the outstanding technological advancement and creativity of the universities, research institutes and industries of the world during these three decades. Also, the journal has been a pioneer and a positive representation of fields of advanced robotics, always providing a wide diversified range of topics that are progressive and full of potential.

As all successes have a cornerstone, let me take this opportunity to once more, emphasize that founding reasons of RSJ were much larger than just the academic sphere; robotics would have an essential part not only in the technological but also in the psychological, social and economic sphere.

KROS strongly shares these goals of promoting the progress of science and technology that will enhance and develop the international community of research, industry and society. We are proud to maintain a close relationship with RSJ. The two societies have collaborated for 10 years since KROS has been established in 2002.

May KROS and RSJ continue to treasure these important values, friendship and be the ground for academic discourse and cooperation.

Congratulations again to the Robotics Society of Japan for its 30th anniversary, to its Board and officers, and to its members for the outstanding achievements and service to their country and the international robotics community. We trust that RSJ's distinguished activities will continue in many years to come. 